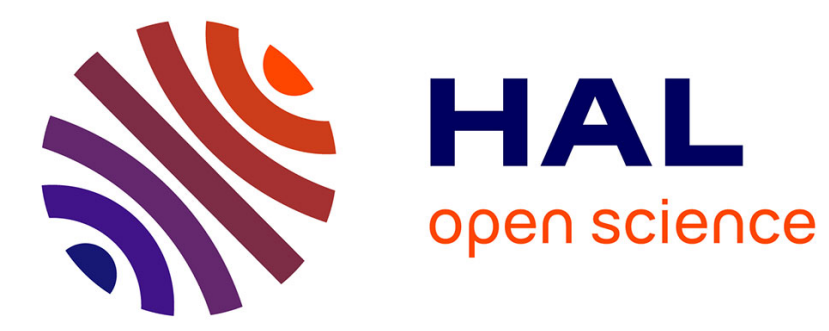

\title{
Les formations de remblaiement des vallées mortes de la presqu'île de Crozon
}

Bernard Hallegouet

\section{To cite this version:}

Bernard Hallegouet. Les formations de remblaiement des vallées mortes de la presqu'île de Crozon. Norois, 1976, 92, pp.615-622. 10.3406/noroi.1976.3544 . insu-02279205

\section{HAL Id: insu-02279205 \\ https://hal-insu.archives-ouvertes.fr/insu-02279205}

Submitted on 17 Feb 2021

HAL is a multi-disciplinary open access archive for the deposit and dissemination of scientific research documents, whether they are published or not. The documents may come from teaching and research institutions in France or abroad, or from public or private research centers.
L'archive ouverte pluridisciplinaire HAL, est destinée au dépôt et à la diffusion de documents scientifiques de niveau recherche, publiés ou non, émanant des établissements d'enseignement et de recherche français ou étrangers, des laboratoires publics ou privés.

\section{(이) $\$$}

Distributed under a Creative Commons Attribution - NonCommercial - NoDerivatives $\mid 4.0$ 
Les formations de remblaiement des vallées mortes de la presqu'île de Crozon

Bernard Hallégouët

Citer ce document / Cite this document :

Hallégouët Bernard. Les formations de remblaiement des vallées mortes de la presqu'île de Crozon. In: Norois, $n^{\circ} 92$, Octobre- $^{2}$ Décembre 1976. pp. 615-622;

doi : https://doi.org/10.3406/noroi.1976.3544

https://www.persee.fr/doc/noroi_0029-182x_1976_num_92_1_3544

Fichier pdf généré le 09/11/2019 


\title{
LES FORMATIONS DE REMBLAIEMENT des VAllées mortes de LA PRESQU'Ile de CROZON
}

\author{
par Bernard HALlEGoUË̈
}

Le relief de la presqu'île de Crozon se caractérise par une alternance de sillons et de crêtes appalachiennes dont les sommets aplanis forment des plateaux en lanières qui s'abaissent jusque vers 50 mètres d'altitude. Quelques dépressions traversent de part en part la presqu'île et plusieurs auteurs les ont interprétées comme étant d'anciennes vallées abandonnées à la suite de captures. Ce sont du Nord au Sud :

- la vallée de l'étang de Kervian au Sud de Roscanvel,

- la dépression de Quélern située à la base de la presqu'ile de Roscanvel entre l'anse de Camaret et la baie de Roscanvel,

- la vallée comprise entre les anses du Fret et de Dinan,

- la vallée de l'Aber s'allongeant entre l'Aulne et l'anse de Morgat,

- la dépression de Lostmarc'h à l'Ouest de Morgat.

A. Vacher (1919) pensait que la vallée du Fret, où la ligne de partage des eaux se situe vers 28 mètres d'altitude, avait été empruntée par l'Aulne et que la dépression de Quélern avait été creusée par un cours d'eau provenant de la presqu'île de Plougastel. L. Collin (1923-1926) voyait dans la dépression de Lostmarc'h et la vallée de l'Aber de part et d'autre de l'anse de Morgat, un autre cours abandonné de l'Aulne. Au Nord de la presqu'île de Crozon, l'étude des photographies aériennes de la région de Roscanvel a montré que la vallée de l'étang de Kervian est une vallée morte dont le cours supérieur a été capturé par le ruisseau de la Fraternité (Hallégouët, 1971), mais aucune formation marine ou fluviatile ancienne n'apparaît au fond de cette dépression. Cependant des dépôts, dont certains avaient été signalés par $A$. Vacher et $L$. Collin, subsistent dans les autres vallées de la presqu'île ; mais ils n'avaient jamais été étudiés à part ceux de Trez-Rouz (Melou, Morzadec).

\section{Les dépôts.}

\section{La dépression de Quélern.}

Le fond de l'isthme déprimé de Quélern qui culmine vers 13 mètres, est occupé par des formations périglaciaires, où sont interstratifiés des dépôts interglaciaires visibles en coupe dans les falaises de l'anse de Trez-Rouz. L'analyse pollinique a permis d'y distinguer deux interglaciaires. L'interglaciaire supérieur a été assimilé à l'Eemien du Nord-Ouest de l'Europe 
continentale et l'interglaciaire inférieur a été rattaché à l'Holsteinien. Ils sont séparés par les coulées de solifluxion d'une phase froide qui serait l'équivalent du Saalien (Morzadec-Kerfourn, 1974). Dans les dépôts interglaciaires inférieurs, la présence des Chénopodiacées et des Hystrichosphères indique la proximité d'un rivage marin. Les sables qui s'y trouvent intercalés renferment un fort pourcentage de tourmaline et de grenat, mais pas de zircon à l'inverse de la coulée de solifluxion inférieure où ce minéral est très abondant (Melou, 1968). Un autre niveau de sable bien classé apparaît dans les dépôts interglaciaires supérieurs. Il est surmonté d'une argile



FiG. 1. -Répartition des dépôts marins ou fluviatiles anciens dans la presqu'ile de Crozon el à ses abords.

1 : cote d'altitude,

2 : rupture de pente convexe,

3 : réseau hydrographique primitif,

4 : capture,

5 : alluvions anciennes de l'Aber,

6 : terrasses de l'Aulne,

7 : dépôts bas-normanniens,

8 : depôts haut-normanniens,

9 : formations de Trez-Rouz,

10 : dépôts marins du rivage de 35 mètres,

11 : formations marines comprises entre 35 et 200 mètres.

grise qui contient une végétation herbacée caractéristique d'un type d'étang très fréquent le long des côtes au niveau des plus hautes mers. Aux deux phases tempérées de Trez-Rouz correspondent donc deux transgressions marines qui ne semblent guère avoir dépassé l'altitude de 6-8 mètres audessus du niveau moyen actuel. Mais, à la base de la coupe, dans les dépôts 
périglaciaires contemporains de l'Elsterien apparaissent aussi quelques galets de quartz très bien émoussés. Ils proviennent sans doute du remaniement de dépôts marins plus anciens dont un gisement subsiste dans la partie orientale de l'isthme près de Pen-ar-Poul-Trémet vers 25 mètres d'altitude.

Ce dépôt en partie consolidé par les oxydes de fer, est formé de galets et de graviers de quartz ou de quartzite, englobés dans une matrice sableuse dont une fraction importante des grains a subi une évolution éolienne. Mais les galets de quartz offrent tous les caractères d'une formation marine [ médiane des émoussés : $312(2)$ ]. L'ilménite, la tourmaline et la staurotide sont les minéraux lourds les plus fréquents dans les sables. Les zircons y sont peu abondants.

L'abrupt situé au Sud de Pen-ar-Poul-Trémet et se poursuivant vers l'Est jusqu'au Fret peut ètre interprété comme une falaise morte. Un sondage à la tarière implanté à son pied entre Kervéguen et Kérifloc'h vers 30 mètres d'altitude, a révélé la présence d'un sable fin argileux sous deux mètres de formations limoneuses englobant des cailloux et quelques galets de quartz. Le sable comprend dans sa partie supérieure une forte proportion de grains éolisés. Les minéraux opaques (ilménite, monazite grise) y sont abondants et représentent $33 \%$ de la fraction lourde. Le zircon, le rutile et la tourmaline forment $55 \%$ des minéraux lourds transparents tandis que les minéraux de métamorphisme (andalousite, staurotide et disthène) en composent $40 \%$. On a noté d'autre part l'absence de grenat et la rareté de la glauconie. Fn profondeur le sable devient de plus en plus graveleux et, vers 3 mètres, il renferme de nombreux galets.

\section{La vallée du Fret.}

Cette vallée qui s'ouvre entre l'anse du Fret et l'anse de Dinan est en partie remblayée par des sables et des galets dont les dépôts les plus importants affleurent près de l'étang de Kerloc'h. Ils apparaissent en coupe sur les rives de celui-ci à proximité de l'ancienne gare de Perros. Les galets sont formés de quartz, de grès armoricain, de quartzite gedinnien, de schiste gréseux el de grès du Siegenien inférieur et sont bien émoussés. Vers la base des coupes, des galets fantômes sont réduits à l'état d'argile ou de sable, et quelques galets de grès armoricain sont aussi altérés que ceux du gisement du Ménez-Luz en Telgruc. Entre les niveaux de galets emballés dans une matrice gravelo-sableuse, s'intercalent des lentilles de sable parfois bien trié [So $=1,50(3)]$ où l'on remarque une assez forte proportion de grains ayant subi une évolution marine. Les minéraux opaques (ilménite, magnétite, limonite, monazite grise) représentent $39 \%$ de la fraction lourde. Dans les minéraux transparents, les minéraux de métamorphisme sont particulièrement abondants : $58 \%$, dont $42 \%$ d'andalousite. On compte par ailleurs, $17 \%$ de tourmaline, $12 \%$ de zircon et $8 \%$ de rutile. Les autres minéraux identifiés sont l'anatase, la monazite et l'amphibole.

Les dépôts affleurant en contrebas du Restou vers 15 mètres d'altitude sont situés stratigraphiquement au-dessus de ceux de la gare de Perros. Les galets de quartz sont bien émoussés ( $M d=387$ ) et les grès et les quartzites, à l'inverse de ceux de la coupe de Perros, ne sont pas altérés. Les sables grossiers ou graveleux formant leur matrice comprennent des grains ayant

(2) Indice d'émoussé : $\frac{2000 \mathrm{R} 1}{\mathrm{~L}}$
(3) So $: \sqrt{\frac{\mathrm{Q3}}{\mathrm{Q1}}}$ 
subi une évolution marine, mais les grains éolisés sont les plus abondants. Comme à Perros, la base de ces formations n'est pas visible et il est probable qu'elles remblaient en partie le fond de l'étang de Kerloc'h.

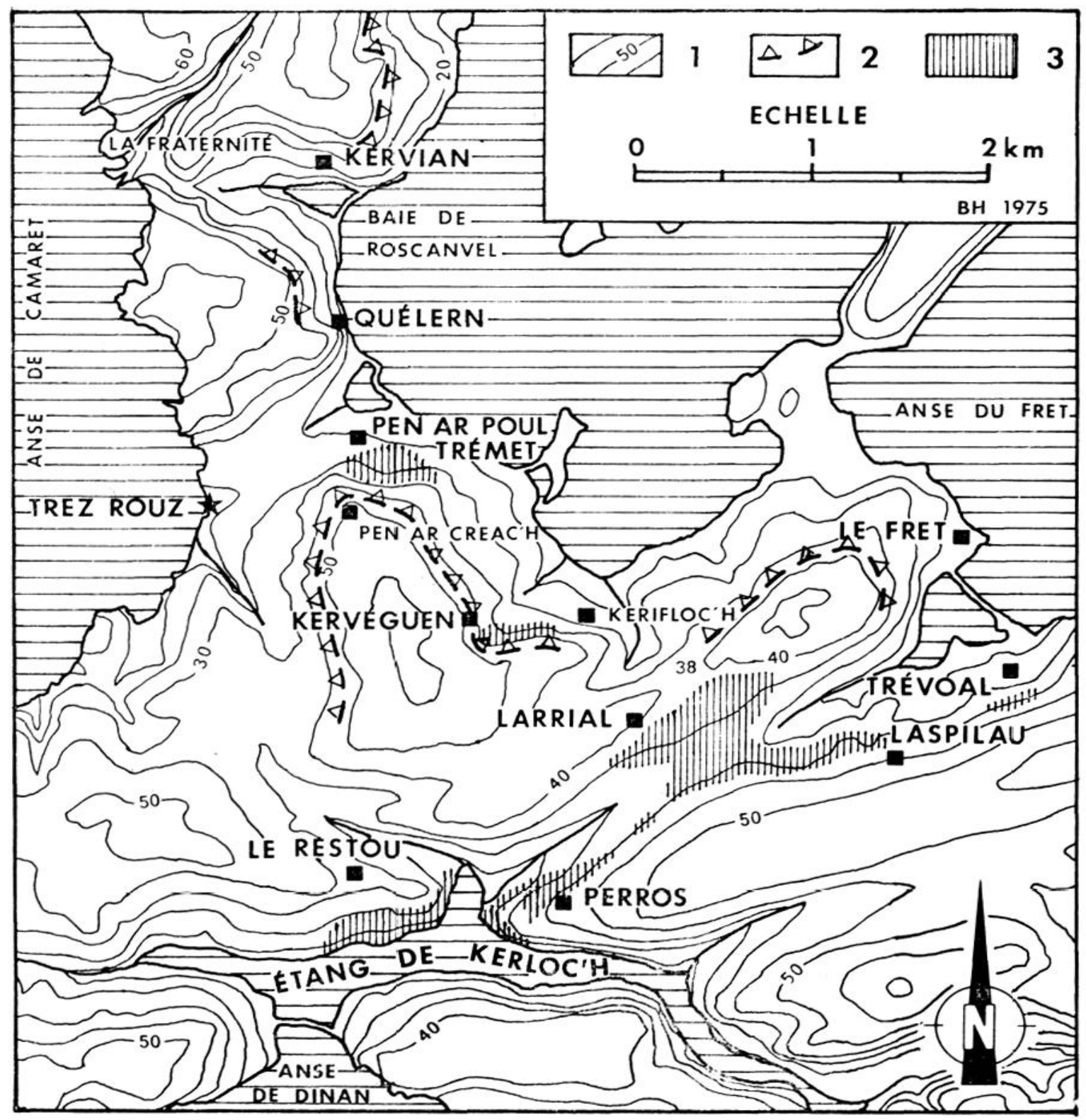

Fici. 2. - Localisalion des formations marines

dans la vallce du Fret el la dépression de Quélern.

1 : courbes de niveau,

2 : falaise morte,

3 : formations marines appartinant au rivage de $3 \overline{5}$ metres.

Sur le versant au Nord du village de Perros, des galets apparaissent jusqu'à 35) mètres d'altitude. Plus à l'list vers le même niveau, des sables fins englobant quelques petits galets de quart\% affleurent au fond de tranchées de drainage à proximité de Iarrial et de part et d'autre de la route de Crozon à Roscanvel. Les coupes du versant sud présentent des poches de galets et de sable formant parfois des poudingues ferrugineux. Les galets situés à la base de la formation sont en général assez gros, peu émoussés et formés 
surtout de quartzite, tandis que ceux qui apparaissent au sommet sont de petite taille, bien émoussés et en grande partie formés de quartz. Le matériel sableux provient presque essentiellement de la désagrégation des grès friables du Siegenien inférieur formant le substratum, mais certains niveaux qui se caractérisent par la finesse du grain et un meilleur classement (So = $1,171)$ possèdent de nombreux éléments ayant subi une évolution marine. A défaut de sondage, l'épaisseur du remblaiement dans la partie centrale de la dépression n'a pu être précisée. Vers l'anse du Fret, les dépôts sont peu abondants et les poudingues et les grès ferrugineux affleurant vers Trévoal entre 30 et 35 mètres d'altitude possèdent les mêmes caractères que les autres dépôts de la vallée.

\section{La vallée de l'Aber et la dépression de Lostmarc'h.}

Aucun dépôt comparable à ceux des vallées du Fret et de Quélern n'a jusqu'à présent été découvert dans la vallée de l'Aber. Mais dans la tranchée rectifiant le cours du ruisseau installé au fond de la dépression, des alluvions anciennes composées de galets de grès, de quartzites et de quartz mal roulés apparaissent sous des limons périglaciaires. Ces alluvions qui sont cryoturbées et forment parfois des poudingues ferrugineux, reposent sur des formations périglaciaires plus anciennes, souvent rubéfiées. Aucun dépôt marin ou fluviatile n'apparaît sous les formations périglaciaires remblayant le fond de la vallée de Lostmarc'h. Son abandon par le ruisseau de l'Aber à la suite de l'ouverture de l'anse de Morgat semble très ancien.

A l'Est du seuil d'Argol, l'anse de Garo a été en partie remblayée par des galets fluviatiles dont la mise en place semble contemporaine de l'édification des terrasses de l'Aulne. Ces dernières qui apparaissent au-dessus des rives convexes des méandres ne semblent pas dans l'estuaire s'élever à une altitude supérieure à 25 mètres. Leur matériel se compose surtout de galets de grès ou de quartz assez émoussés et la matrice argileuse ou sableuse les englobant est souvent rubéfiée.

\section{Interprétation paléogéographique.}

Les alluvions de la vallée de l'Aber ne présentent aucun point commun avec celles de l'Aulne et le seuil d'Argol, à 62 mètres, se situe bien au-dessus de l'altitude atteinte par les terrasses de l'Aulne dans son estuaire. Il paraît donc exclu qu'à une époque récente, l'Aulne ait emprunté le seuil d'Argol pour s'écouler en direction de la dépression de Lostmarc'h.

Les anciennes formations de remblaiement des vallées mortes du Nord de la presqu'île de Crozon ne correspondent pas non plus aux alluvions de l'Aulne, mais à des dépôts marins culminant généralement vers 35 mètres d'altitude. Ces dépôts sont azoïques et aucun indice ne permet de les dater de mème que ceux du Ménez-Luz se situant vers 125 mètres (Guilcher et Saint-Requier, 1969) ou ceux de la vallée de l'Elorn apparaissant à des altitudes intermédiaires (Hallégouët, 1976). Cependant par analogie avec certaines formations de sables rouges de la partie orientale du Massif Armoricain ou avec les dépôts marins de la surface de 430 pieds de l'Ouest du Cornwall, les galets du Ménez-Luz sont habituellement considérés comme pliocènes. L'altération du grès armoricain dans la partie inférieure de la coupe de Perros suggère que les dépôts remblayant le fond de l'étang de Kerloc'h sont également très anciens. Le cortège des minéraux lourds qui y apparaissent se différencie nettement de celui des sables holsteiniens de Trez-Rouz ou de celui des sables haut-normanniens de Goulven sur le littoral septentrional du Léon (Hallégouët, 1971), du fait de l'absence de grenat. 
Ce caractère et l'abondance des minéraux de métamorphisme permettent un rapprochement avec les sables pliocènes affleurant près de Toulven au Sud de Quimper (Melou, 1968). D'autres formations marines apparaissent aussi vers 30-35 mètres d'altitude dans le Nord de la Bretagne et en Cornouaille britannique. Celles de Pont-Rouz dans la vallée du Guindy (Trégor) ont été attribuées au Reuverien terminal (Morzadec, 1975), mais l'âge de celles de Saint-Frth au Nord-Est de Penzance (Cornwall) (4) d'abord considérées comme pliocènes, est encore discuté (Mitchell, 1965). Les dépôts s'échelonnant entre 4 et 35 mètres dans la vallée du Fret ne sont peut-être pas tous du même âge, mais ils semblent cependant devoir être rangés dans le Pliocène. Dans la vallée de l'Elorn, la mer paraît cependant avoir atteint pendant le Quaternaire, l'altitude de 35 mètres, mais ce ne fut qu'un épisode très bref (Hallégouët, 1976) et aucune formation de ce type n'a été trouvée dans la presqu'île de Crozon.

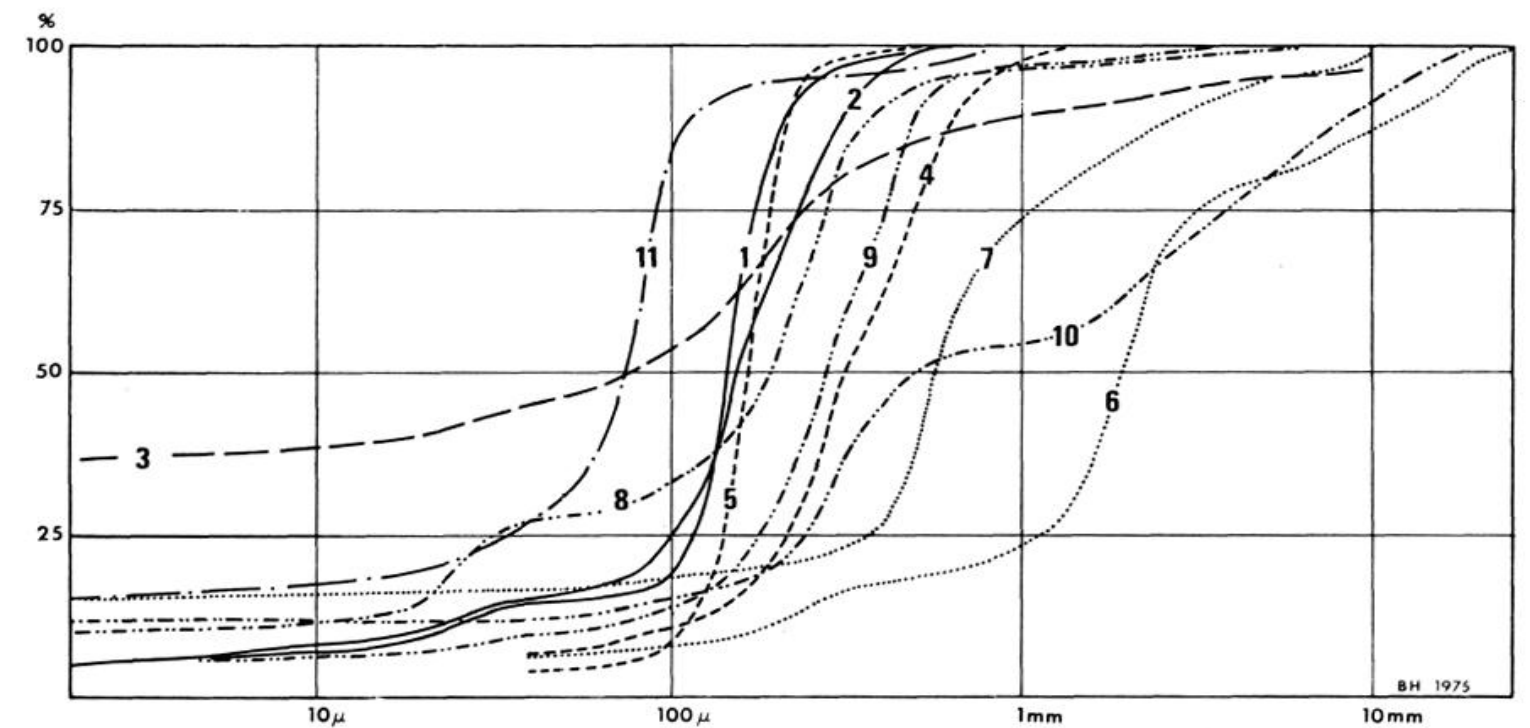

Fı́. 3. - Courbes granulométriques des dépôts sableux.

1 : Camaret-Trez Rouz : sable émien,

2 : Camaret-Trez Rouz : sable holsteinien,

3 : Crozon-Kervéguen : niveau supérieur,

4 : Crozon-Laspilau : sable provenant de la désagrégation des grès siegeniens,

5 : Crozon-Laspilau : niveau bien remanié par la mer,

6 : Camaret-Le Restou : niveau graveleux à la base de la coupe,

7 : Camaret-Le Restou : niveau sableux vers le sommet de la coupe,

8 : Crozon-Perros : niveau sableux à la base de la coupe,

9 : Crozon-Perros : niveau sableux au milieu de la coupe,

10 : Crozon-Perros : sable graveleux de la partie supérieure de la coupe,

11 : Telgruc-Ménez Luz: accumulation sableuse au Nord-Ouest de la butte.

La position des dépôts montre que lors de sa dernière submersion par la mer, la vallée du Fret était déjà abandonnée par la rivière qui l'avait creusée. Il s'agissait vraisemblablement de la rivière de Kerloc'h qui devait s'écouler en direction de la rade de Brest avant sa capture par le ruisseau qui drainait l'emplacement de l'anse de Dinan. On peut se demander si la vallée de Quélern n'a pas eu également pour origine un cours d'eau descendant des hauteurs de Camaret et se dirigeant vers le Nord-Est. Cette vallée aurait été ensuite tronquée par l'érosion marine lors du creusement

(4) Les sables de ce dépòt se différencient nettement de ceux des vallées mortes de la presqu'ile de Crozon par le fait qu'ils ne renferment qu'une faible proportion de grains éolisés. Ils sont aussi très riches en glauconie. 
de l'anse de Camaret. Cette disposition du réseau hydrographique primitif s'explique par le fait que l'emplacement de la rade de Brest était alors une cuvette dont la vallée Penfeld-Aber Ildut assurait l'écoulement des eaux vers la mer (Hallégouët, 1971) (5). Ce réseau hydrographique fut ensuite démantelé par les cours d'eau drainant l'emplacement de l'Iroise et la rivière du goulet de Brest plus favorisée, car travaillant dans la bande des quartzophyllades de l'Elorn, captura successivement l'Aulne et l'Elorn.

Au moment où le rivage marin se stabilisa vers 35 mètres, le goulet de Brest devait ètre déjà profondément creusé. Aussi les courants parcourant les passes de Quélern et du Fret étaient affaiblis et une sédimentation marine put s'y développer. Cependant la position d'abri du dépôt de Pen-arPoul-Trémet à l'Est de la passe de Quélern semble indiquer que les courants de marée étaient encore assez forts dans ce passage. La mer submergea de nouveau l'isthme de Quélern au cours du Quaternaire et en particulier lors de la transgression qui forma les plages haut-normanniennes du littoral septentrional du Pays de Léon (6) et celle de la pointe de Saint-Hernot dans la presqu'île de Crozon. Cet épisode transgressif, d'abord placé dans l'Eemien, tend depuis quelques années à être rattaché à l'Holsteinien par une partie des auteurs (Giot et Monnier, 1972). Il pourrait aussi ètre plus ancien, car si la mer avait envahi l'isthme de Quélern à l'Holsteinien, les courants de marée auraient pu déblayer les dépôts périglaciaires inférieurs de TrezRouz ou les auraient tout au moins ravinés en y laissant quelques traces. Or rien de tel n'apparaît et les formations comblant un chenal creusé dans les coulées périglaciaires de l'Elsterien sont d'origine lacustre. Comme les tourbes et les sables marins reposant sur ces dépôts correspondent à un épisode transgressif, qui ne dépassa pas de beaucoup l'altitude du niveau marin actuel, il est possible qu'une période froide correspondant aux dépôts périglaciaires inférieurs de Trez-Rouz ait existé entre la transgression du Haut-Normannien et la mise en place des formations holsteiniennes.

\section{Conclusion.}

Les vallées mortes de la presqu'île de Crozon sont des dépressions appalachiennes creusées dans des bandes de roche tendre ou des synclinaux faillés. La vallée Aber-Lostmarc'h n'a vraisemblablement jamais été empruntée par l'Aulne, et celles du Fret, de Quélern et de Kervian sont des tronçons de vallées abandonnées dont le drainage était orienté autrefois vers la rade de Brest. Les dépôts d'un rivage marin qui se stabilisa aux alentours de 35 mètres vers la fin du Pliocène, se sont conservés dans les dépressions du Fret et de Quélern. Par la suite, au cours du Quaternaire, ce niveau ne semble pas avoir été dépassé. A Trez-Rouz, à l'Holsteinien, la mer ne paraît pas avoir monté beaucoup au-dessus de son niveau actuel et il n'est pas exclu que les dépôts du Haut-Normannien représentés par les galets de la pointe de Saint-Hernot dans la presqu'île de Crozon, correspondent à une phase tempérée antérieure.

\section{REFÉRENCES BIBLIOGRAPHIQLES}

Col.tiv L., 1923. Un ancien cours de l'Aulne. Bull. Soc. Géol. Minér. Bretagne, 4, 3, p. 294.

Coll.t. L., 1926. La dépression de Morgat à Lostmarc'h. Ancien passage de l'AulneAber. Bull. Soc. Géol. Minér. Brelagne, 7, 1-2, pp. 135-138.

(5) Ceci a été confirmé par la découverte d'énormes quantités de galets formés du grès armoricain de la presqu'île de Crozon au fond de cette vallée à l'Ouest de Guilers.

(6) Ces plages culminent vers 23 mètres au-dessus du niveau moyen. 
Collin L., 1936. Formations quaternaires de l'Ouest du Finistère. Bull. Soc. Géol. et Minér. Brelagne, n. série Fasc. Spécial 69 p.

Guifcher A. et Saint-Requier A., 1969. Les galets et les sables du Ménez Lus (Finistère), et leur signification morphologique. Norois, n 62, pp. 245-251.

Giot P. R. et Monvier J. L., 1972. Chronologie relative des plages anciennes, des industries, des limons et des sols fossiles associés du Nord de la Bretagne. C.R. Acad. Sc. Paris, t. 274, série D, pp. 2288-2290.

Hallegouet B., 1971. Le Bas-Léon (Finistère). Élude géomorphologique. 'Thèse $3^{\mathrm{e}}$ cycle, Brest, 260 p., 87 fig., 17 pl. hors texte.

Hallegouet B., 1976. Les anciens dépôts marins et fluviatiles de la vallée de l'Elorn (Finistère). Norois, no 89 , pp. 55-72.

Kerfours M. T., 1965. Le dépòt tourbeux de l'anse de Trez Rouz à Camaret (Finistère) peut être rapporté à l'interglaciaire Mindel-Riss. C.R. Acad. Sciences de Paris, 260, pp. 2024-2026.

Mitchell G. F., 1965. The St-Erth beds. An alternative explanation. Proceedings of the Geologists Association. Vol. 76, Part 4, 1965, pp. 345-366.

Mr.ou M., 1968. Contribulion à l'étude sédimentologique du Quaternaire sud-finistérien. L'Anse de Trez-Rouz el la ria de l'Odet. Orsay, thèse de $3^{\text {e }}$ cycle, 81 pages.

Morzadec-KerfourN M. T., 1969. Ie Quaternaire de la plage de Trez-Rouz (NE) de Camaret dans le Finistère). Bull. Ass. Fr. Et. Quat., 6, 19, 2, pp. 129-138.

MoRzadec-K ERFourN M. T., 1974. Varialion de la ligne de rivage armoricaine au Quaternaire. Analyses polliniques des dépôts organiques litloraux. Thèse, Rennes, $208 \mathrm{p}$.

Morzanec-Kerfourn M. T., 1975. Le Plio-Quaternaire marin de Pont-Rouz iCóles du Nord). C.R. Acad. Sc. de Paris, t. 280, série D, pp. 1953-1957.

Musset R., 1934. Ia formation du réseau hydrographique de la Bretagne Occidentale. Ann. Géogr., 43, pp. 561-578.

VACHER A., 1919. La rade de Brest et ses abords, essai d'interprétation morphogénétique. Ann. Géogr., 28, pp. I77-207. 